\title{
Abondance et précarité. Conditions de vie et alimentation des sans-abri à Tahiti
}

\section{Christophe Serra Mallol}

\section{(2) OpenEdition}

12 Journals

Édition électronique

URL : http://journals.openedition.org/jso/5824

DOI : 10.4000/jso.5824

ISSN : $1760-7256$

Éditeur

Société des océanistes

\section{Édition imprimée}

Date de publication : 15 décembre 2009

Pagination : 263-278

ISBN : 978-2-85430-026-0

ISSN : 0300-953x

Référence électronique

Christophe Serra Mallol, « Abondance et précarité. Conditions de vie et alimentation des sans-abri à Tahiti », Journal de la Société des Océanistes [En ligne], 129 | juillet-décembre 2009, mis en ligne le 30 décembre 2012, consulté le 21 avril 2019. URL : http://journals.openedition.org/jso/5824 ; DOI $10.4000 / j s 0.5824$

\section{(c) Tous droits réservés}




\title{
Abondance et précarité. Conditions de vie et alimentation des sans-abri à Tahiti
}

par

\author{
Christophe SERRA MALLOL*
}

\section{RÉSUMÉ}

Malgré l'image de paradis terrestre, d'abondance alimentaire et de relations sociales basées sur l'hospitalité dont fait l'objet Tahiti, et la manne des transferts publics déversée sur le pays depuis une quarantaine d'années, l'agglomération de Papeete accueille pourtant un nombre non négligeable de personnes vivant dans la rue. Une enquête exhaustive a été menée auprès de cette population, pour mieux cerner ses conditions socioéconomiques de vie, notamment en matière alimentaire. Nous nous poserons la question de l'influence réciproque des représentations traditionnelles de Tahiti et de celles concernant les personnes sans-abri sur la prise en compte d'une réalité vécue dans la rue, et sur ces personnes elles-mêmes.

Mots-CLÉs: Tahiti, Polynésie française, sans-abri, exclusion, alimentation

La Polynésie française et, en particulier, Tahiti ont fait et continuent à faire l'objet d'une vision édénique créée par les premiers Européens à la fin du XVIII ${ }^{\mathrm{e}}$ siècle : climat paradisiaque et abondance naturelle, relations sociales dépourvues de conflit fondées sur un communautarisme partageux... De la nourriture rendue disponible par la nature sans aucun effort humain et offerte par brassées par des indigènes édéniques à leurs visiteurs : le mythe de l'abondance et de l'hospitalité

\section{ABSTRACT}

In spite of the image of paradise on Earth, food abundance and social relationships based on the hospitality of which Tahiti is the object, and the considerable amount of the public transfers poured since about forty years, the urbanized zone of Papeete welcomes nevertheless an important number of persons living in the street. An exhaustive survey has been led with this population, to identify better its socioeconomic conditions of life, in regard to food in particular. We shall ask the question of the mutual influence of the traditional representations of Tahiti and of those concerning the homeless persons on apprehending a reality lived in the street, and on these very persons.

Keywords: Tahiti, French Polynesia, homeless, exclusion, food tahitiennes était né (Serra Mallol, 2005). Ces représentations se sont diffusées dans l'ensemble du monde occidental, et ont été intégrées par les acteurs locaux eux-mêmes.

Avec l'implantation du Centre d'expérimentation du Pacifique (CEP) au début des années 1960, la Polynésie française est entrée dans la modernité et la croissance économique. Cette période de constructions militaires et civiles sans précédent a attiré vers le salariat un grand nombre

* Socio-anthropologue, enseignant-chercheur à l'Institut supérieur de l'Enseignement privé de Polynésie française (ISEPPUCo), csm@mail.pf. 
d'agriculteurs-pêcheurs résidents des îles éloignées, parmi les plus jeunes, leur permettant de sortir d'une vie confinée à l'autosubsistance avec l'espoir d'accéder à une autonomie financière, à la vie urbaine, aux sorties, aux objets de consommation, aspirations à une vie quotidienne transformée dans tous ses aspects (Fagès, 1973, 1974). Mais cette croissance extraordinaire au cours des quarante dernières années a creusé les inégalités socio-économiques, provoquant la création d'un prolétariat urbain (Lockwood, 2002) et d'une frange relativement importante d'exclus, et notamment de personnes sans-abri. C'est dans ce contexte particulier qu'il nous a paru intéressant de mener une étude sur ce dernier groupe. Dans le présent article, nous n'aborderons pas les questions des raisons qui poussent à vivre dans la rue, ni la géographie des différents lieux où vivent et dorment les sans-abri. Nous nous limiterons à présenter la réalité vécue sur le terrain par les personnes vivant dans la rue en matière de conditions de vie et notamment d'alimentation et les représentations qu'elles ont de leur situation.

\section{Un groupe particulier : les sans-abri}

\section{Définition de l'objet et méthodes d'enquête}

Face à la multiplicité des termes utilisés (sansabri, sans domicile fixe ou « SDF », personnes en situation d'errance...) et donc de définitions, la population visée par l'étude a été définie comme suit :

- personnes dépourvues d'un espace privé nécessaire à l'accomplissement de fonctions vitales : se nourrir, se vêtir, se laver... dans l'agglomération de Papeete (de la commune de Papara à l'ouest de Papeete à la commune de Mahina à l'est) et à Moorea ;

- personnes fréquentant les structures d'accueil et d'assistance existantes ;

- personnes accueillies à un moment donné dans une structure d'accueil temporaire.

Sont donc exclues du cadre de la présente étude les personnes résidant dans un abri de fortune sur un terrain leur appartenant, ainsi que les familles logées temporairement en attente d'être relogées par décision administrative.

Les éléments recueillis au cours de la phase exploratoire de l'enquête nous conduisent à poser la question de la pertinence de la dénomination « SDF » utilisée au lancement de l'enquête, et telle qu'elle apparaît dans certains documents consultés localement. Ce terme a toujours été ressenti par les personnes rencontrées comme dépréciatif, correspondant à la perception qu'elles avaient du "clochard», «à la française » qui plus est, et à ses signes jugés comme distinctifs : toujours en état d'ébriété, sale et déguenillé, âgé et en mauvaise santé. Elles renvoyaient d'ailleurs cette dénomination aux rares sans-abri polynésiens correspondant à cette description et caractérisés par une "chronicisation » de leur situation (Damon, 2002), ou au traditionnel «hombo» tahitien. Si le terme " SDF » est perçu par les personnes rencontrées comme caractérisant une forme de laisser-aller et de situation de dérive sans retour ('óere), il est surtout ressenti comme une absence d'ancrage inacceptable pour un Tahitien pour qui la terre, le fenua, symbolise l'identité individuelle (l'étranger est communément appelé hutu painu, à l'image de ce fruit qui flotte sur l'eau et dérive au gré des courants).

Les personnes elles-mêmes se désignaient comme "vivant dans la rue » ou " sans-abri », faisant le constat objectif d'une absence de logement, alors que les éducateurs de rue les désignaient plutôt sous le vocable de « sans-abri » ${ }^{1}$ ou « en situation d'errance », mais cette dernière expression peut être perçue de façon péjorative. Ce sont donc indifféremment les dénominations « sans-abri » et « personnes vivant dans la rue » que nous utiliserons ici.

Pour mieux connaître ce groupe et ses conditions de vie, notamment en matière alimentaire, nous avons mené auprès de cette population une étude ${ }^{2}$ à la fois qualitative et quantitative. La partie qualitative a été menée à partir de récits de vie auprès de vingt personnes vivant dans la rue, choisies en raison de caractéristiques sociodé-

1. Même si on peut considérer de façon stricte que la personne « sans-logis » est celle qui se caractérise par une absence de logement, alors que celle « sans-abri » a été victime d'une catastrophe. Mais le fait d'être rejeté par sa famille, ou de fuir des problèmes familiaux jugés insurmontables, peut être vécu par la personne comme une « catastrophe sociale ».

2. Cette étude, commanditée par le Contrat urbain de cohésion sociale (CUCS) de l'agglomération de Papeete au sein duquel je remercie Sylvie Jarles et Heimana Ah-Min, s'est déroulée entre novembre 2007 et septembre 2008 en trois phases : une phase documentaire et d'entretiens qualitatifs approfondis auprès de vingt sans-abri, une phase d'enquête quantitative de type exhaustif par recensement systématique auprès de plus de trois cent cinquante sans-abri dans l'agglomération urbaine de Papeete et l'île de Moorea, et une dernière phase d'évaluation des structures d'aide existantes et d'entretiens auprès d'acteurs associatifs et institutionnels. Les résultats complets de l'enquête ont été présentés en septembre 2008 devant les principaux représentants des communes, du pays et de l'État en charge des questions d'exclusion sociale et économique. Des travaux de mise en œuvre d'actions correctives et préventives sont en cours à partir d'un plan d'action global proposé par l'auteur, et une évaluation continue du plan d'action est prévue au cours des deux années à venir. 
mographiques variables (âge, sexe, situation familiale, durée de vie dans la rue). Il a fallu souvent deux à trois contacts avant d'aboutir à un entretien complet et exploitable. Les entretiens ont été menés en grande majorité dans la rue, tout au long de la journée (de 05h30 du matin à 21 h00 le soir) et tous les jours de la semaine, samedi et dimanche compris, pendant les trois dernières semaines du mois de novembre 2007.

Les entretiens de la phase quantitative, d'une durée comprise entre vingt et quarante-cinq minutes, ont été conduits en quasi-totalité dans la rue, dans les principaux « spots » et lieux de vie des sans-abri, en matinée surtout (de $5 \mathrm{~h} \mathrm{du}$ matin à 13h), du 13 au 21 décembre 2007, et du 7 janvier au 5 février 2008. Quelques entretiens ont été menés le soir à l'occasion des repas gratuits de début de mois, et une minorité dans les centres d'hébergement. Des questionnaires ont également été remplis directement dans quelques "squats" ou "châteaux", après prise de rendez-vous préalable avec les différents occupants.

Nous ne traiterons pas ici des difficultés rencontrées en cours d'enquête inhérentes à la population visée par l'étude, des représentations de l'enquêteur lui-même et de leur influence sur le phénomène observé, ni des interactions inévitables entre deux groupes de population aux caractéristiques socio-économiques différentes entrant en contact (Bourdieu, 1993), qui ont fait l'objet d'un développement par ailleurs (Serra Mallol, 2008).

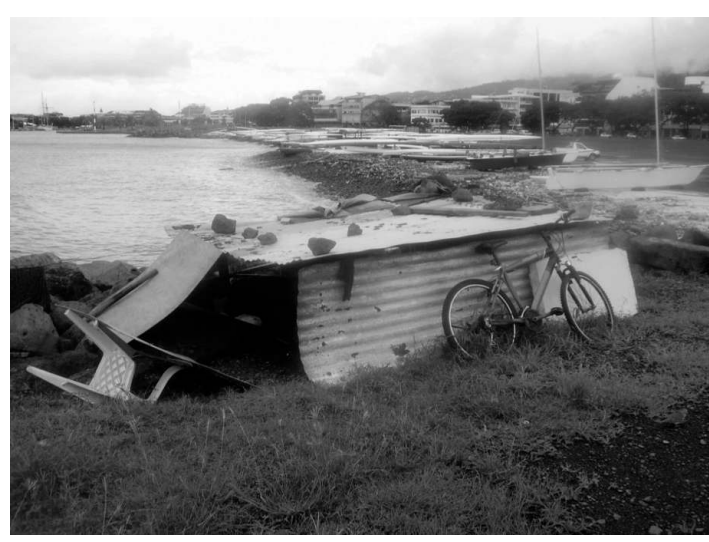

Рното 1. - « Squat » ou cabane fait de matériaux de récupération (cliché de l'auteur)

Parmi l'ensemble des acteurs associatifs et institutionnels rencontrés ${ }^{3}$, les élus communaux et du pays ou leurs représentants ${ }^{4}$ avec lesquels ont eu lieu de nombreux échanges lors des réunions de travail et des présentations des différentes phases de l'étude, nous ne prendrons en compte ici que les réactions des "acteurs de terrain ", constitués par les structures d'aides associatives de proximité et les personnels des services du pays en contact direct avec la population étudiée (Santé, Affaires sociales, Prévoyance sociale, Emploi et Formation, Logement).

\section{L'évaluation quantitative de la population visée}

Le nombre total de personnes interrogées au cours de l'enquête est de 321 personnes $^{5}$ dont $80 \%$ ont été recensées dans la seule ville de Papeete ${ }^{6}$, non compris les 28 enfants de moins de quinze ans vivant avec leurs parents. Nous avons

3. Les structures associatives rencontrées (a minima le responsable et l'adjoint de la structure, sinon les personnels en charge des publics) ont été les suivantes : le Club de prévention spécialisée de l'association Te Torea, le Centre d'accueil Te Vaiete du Secours catholique polynésien, les structures d'hébergement qui dépendent de l'association Emauta (Le Bon Samaritain pour les hommes adultes, la Samaritaine pour les femmes adultes, le Centre Te Arata pour les familles, le foyer maternel Maniniaura pour les jeunes mères célibataires), le Centre d'hébergement du Bon Pasteur destiné aux adolescentes, le Centre d'hébergement Uruai a Tama pour les adolescents, les associations de réinsertion par l'économique Te Rima Turu (Moorea) et Te U'i Rau (Fa'a'a). Seules les cinq premières structures associatives citées œuvrent de façon majoritaire auprès des sans-abri. Les acteurs institutionnels rencontrés ont été les médecins et infirmières des dispensaires communaux de Papeete et de Moorea, les travailleurs sociaux de la caisse de Prévoyance sociale (CPS) et de la direction des Affaires sociales (DAs) ainsi que leurs responsables de circonscription administrative, la personne chargée des sans-abri à la commune de Papeete, des cadres du Fonds de développement des archipels (FDA) et du service de Formation et d'Insertion (SEFI), et des responsables des Églises catholique, protestante et adventiste.

4. Et notamment des membres des cabinets ministériels de la Solidarité, du Logement et de la Santé à la vice-présidence, ainsi que les directeurs ou adjoints des directions des services administratifs de tutelle, et les maires ou adjoints des communes concernées par l'étude, soit Papeete, Fa'a'a, Punaauia, Papara, Pirae, Arue, Mahina et Moorea, et leurs chargés de mission auprès du cUCS.

5. Le chiffre total d'entretiens réalisés est de 341, non compris les 42 enfants âgés de moins de quinze ans vivant avec leurs parents, soit 383 personnes au total. Mais le fait que certains entretiens ont été menés auprès de familles accueillies au sein de l'ancien hôpital Vaiami en vue d'un possible relogement ultérieur nous a conduit à les faire sortir du champ de l'étude, et donc de l'échantillon total.

6. Contrairement aux affirmations initiales de certains élus ou adjoints communaux de l'agglomération urbaine («nous n'avons pas de sans-abri chez nous »), nous avons pu vérifier que toutes les communes composant l'agglomération urbaine de Tahiti, sans exception, accueillaient des sans-abri sur leur territoire. En revanche, c'est le chef-lieu Papeete qui en rassemble le plus proportionnellement à sa population résidente, du fait de l'attraction que la « capitale » et ses commodités exercent, mais également en fonction d'un phénomène que certains auteurs (Damon, 2002) qualifient de « jeu de ping pong », qui consiste à déplacer le problème (en l'occurrence les sans-abri) chez le voisin en les y envoyant, le Not in My Back Yard des Américains. 
été amenés à faire une différence de situation entre des sans-abri que l'on peut qualifier de «permanents », qui constituent précisément le public suivi à Papeete par l'équipe du Club de prévention spécialisée ${ }^{7}$ et des sans-abri que nous nommerons « temporaires » qui vivaient dans la rue au moment de l'enquête quantitative mais dont les réponses permettent d'indiquer qu'ils font des retours périodiques vers un logement, soit leur logement familial d'origine, soit des logements successifs et temporaires, à considérer donc en termes de « flux ». Parmi les 321 personnes interrogées, environ $55 \%$ constituent des sans-abri « permanents » (environ 170 personnes) et environ $45 \%$ des sans-abri « temporaires » (environ 150 personnes), et 19 d'entre elles vivaient dans la rue avec leurs enfants mineurs (1,5 en moyenne), soit 28 enfants. La population totale prise en compte dans l'étude est donc de 349 personnes.

Mais ce chiffre ne constitue qu'une " photographie » du phénomène pendant le déroulement de l'enquête statistique. En effet, le « flux » observé pendant les six semaines d'enquête quantitative, soit les cent cinquante personnes considérées comme sans-abri "temporaires» dont la présence dans la rue est de six mois en moyenne, se transforme en «flux » de trois cents personnes sur l'année. Sur une année complète, on peut donc estimer qu'environ cinq cents personnes sont à un moment ou à un autre "sansabri », enfants compris. On peut de plus considérer que cette population est sous-estimée ( « fuite» devant les enquêteurs, personnes en mouvement qui n'ont pas été repérées, personnes vivant dans des endroits reculées et inaccessibles dans les conditions de l'enquête, etc.) à $30 \%{ }^{8}$. On aurait donc un total de six cent cinquante personnes sur une année qui représenterait un pourcentage d'un peu plus de $0,4 \%$ par rapport à la population considérée, celle de l'agglomération de Papeete et de l'île de Moorea où résidaient 146716 personnes en $2007^{\circ}$ : nous sommes donc proches des taux moyens relevés pour la France, et bien loin des données officielles disponibles localement, qui évaluent les personnes vivant dans la rue pour l'ensemble de la Polynésie française à environ deux cents personnes, dont $60 \%$ dans la seule agglomération de Papeete $^{10}$.

\section{Un profil spécifique}

\section{Age, sexe, provenance géographique}

En matière de sexe, le profil des 321 personnes interrogées ${ }^{11}$ peut être scindé en trois groupes, selon les répondants eux-mêmes : les hommes constituent $81 \%$ du total ${ }^{12}$, bien plus nombreux que les femmes (14\%), et que les rae$\mathrm{rae}^{13}(5 \%)$. Nous estimons toutefois le nombre de femmes sous-évalué, car la plupart des refus de répondre (verbal, ou par " fuite physique ») provient en majorité de femmes accompagnées d'enfants mineurs, qui tentent ainsi d'échapper à ce qu'elles perçoivent comme un recensement officiel, avec le risque de se voir dessaisir de leurs enfants.

7. Le Club de prévention spécialisée est une structure associative créée en 1998 regroupant onze personnes (en majorité travailleurs sociaux de formation) et dédiée à « l'assistance aux personnes en situation d'exclusion » et aux interventions dans la rue, de jour comme de nuit. En ce sens, on peut l'assimiler à une forme de Samu social polynésien. Un local d'accueil, le « Centre de jour », a été mis à leur disposition en 2002 par la mairie de Papeete ; il est doté d'une douche, d'un lavabo et de toilettes, de machines à laver le linge, de sèche-linge, et de casiers de rangement individuels. L'association dispose depuis 2005 de deux studios et d'un F3 pour loger temporairement des familles.

8. En France métropolitaine, les rapports indiquent que la sous-évaluation est de l'ordre de $100 \%$, et certains ouvrages avancent même des estimations qui varient du simple au quintuple (Zeneidi-Henry, $2002: 38-40$ ). Nous minimisons fortement de façon volontaire notre sous-évaluation du fait de la méthode d'enquête retenue, exhaustive sur le terrain.

9. Selon les premiers résultats statistiques du Recensement général de la Population de 2007 par l'ISPF.

10. Les seules données quantitatives disponibles sont celles du Club de prévention spécialisée, de la circonscription administrative des Affaires sociales de Papeete, et de la commune de Papeete. Les chiffres du Club de prévention spécialisée, les seuls portant sur plusieurs périodes, montrent une augmentation notable du nombre de sans-abri « permanents » identifiés sur le champ géographique d'intervention de l'association, c'est-à-dire la seule commune de Papeete : en 1998-99 le Club en aurait identifié 119, et 130 en 2007. La circonscription des Affaires sociales de Papeete dispose d'une liste de 160 personnes recensées en 2007 comme bénéficiaires d'une aide de la commune sans pour en être forcément originaires, et estime que ces personnes constituent environ les trois quarts de la population totale des sans-abri polynésiens. La commune de Papeete, quant à elle, « estime à deux cents le nombre de SDF à Tahiti, principalement en ville » en 2002 (site internet de la commune).

11. Quelques résultats de la partie quantitative de l'enquête seront comparés à ceux d'une enquête réalisée par l'INED à Paris en 1995 (Firdion et Marpsat, 2000), d'une autre par l'INSEE auprès des usagers de services d'hébergement en janvier 2001 (Brousse, Rochère et Massé, 2002), et d'une enquête menée entre novembre 2003 et janvier 2004 auprès de 585 usagers des structures d'accueil et d'hébergement d'urgence en région Pays de Loire (Fleuret, Pihet et Zeneidi-Henry, 2004).

12. Dans l'enquête INSEE, les hommes représentaient $64 \%$ du total, et $68,5 \%$ du total dans l'enquête Pays-de-Loire. Les chiffres de la présente enquête se rapprochent plus de l'enquête 1995 INED menée à Paris où seuls $17 \%$ des SDF sont des femmes.

13. Un raerae est un homme qui adopte le comportement et l'apparence physique d'une femme, jusqu'à se travestir et recourir à des procédés médicaux (prises d'hormones féminines) ou chirurgicaux (implantation de seins, ablation des organes génitaux masculins). 
En matière d'âge, quatre groupes de taille à peu près équivalente se détachent (sans compter les mineurs de moins de quinze ans vivant avec leurs parents) : les 15-24 ans (23\%), les 25-34 ans $(29 \%)$, les $35-44$ ans $(21 \%)$, les 45 ans et plus ( $27 \%$ ). L'âge moyen est de 35 ans $^{14}$, avec une variation très large (écart-type : 13,6 ans). L'âge moyen auquel on a connu la rue pour la première fois est de 29 ans (écart-type : 11,8 ans), avec des extrêmes qui vont de 10 à 69 ans.

La grande majorité des personnes rencontrées est célibataire (79\%). Le restant vit en concubinage $(14 \%)^{15}$, formant parfois des couples entre sans-abri. Seuls $3 \%$ se déclarent mariés, et $3 \%$ séparés, divorcés ou veufs.

Plus d'un tiers des personnes interrogées $(40 \%)$ ont des enfants mineurs de moins de 15 ans, dont certains qui vivent avec eux dans la rue mais que l'on voit peu, les parents se cachant alors ou n'acceptant que rarement l'entretien. Le nombre moyen d'enfants déclarés est de 2,4 (écart-type : 1,6), dont 1,1 mineur. Sur 115 enfants mineurs ainsi recensés, 28 vivent dans la rue avec les parents, 30 avec l'autre parent, 22 en famille fa' 'a'amu (adoption locale) sans lien familial, 9 placés en famille d'accueil, et le restant dans d'autres situations (chez les grands-parents, oncles et tantes, parrains...).

Pour ce qui concerne le lieu de naissance, on note une part prépondérante de Tahiti $(67 \%)$ et de Papeete ( $57 \%$ ) en particulier, du fait de l'obligation des femmes vivant dans les archipels éloignés dépourvus d'un hôpital d'accoucher à Papeete. Mais l'élément remarquable est la surpondération des personnes provenant des archipels des Australes et des Tuamotu-Gambier (au total $16 \%$ des personnes interrogées) par rapport à leur poids réel dans la population totale $(5,5 \%)$, donnée qui illustre l'importance de l'errance géographique des personnes vivant dans la rue, et qui confirme les phénomènes de migration inter-îles observés depuis les années 1970 vers Tahiti (Lockwood, 1980).

Le lieu de résidence jusqu'à l'âge de 18 ans est variable. Avant l'âge de 12 ans, la grande majorité des personnes interrogées a vécu chez ses parents. Seules trois des personnes interrogées citent la rue comme lieu de vie avant l'âge de 12 ans; $93 \%$ citent un seul lieu de résidence, $3 \%$ en citent $2,1 \%$ en citent 3 ou plus (non réponse : $3 \%$ ). Entre l'âge de 12 et 18 ans, la moitié a vécu chez ses parents naturels, un quart $f a^{\prime} a^{\prime} a m u$, soit adopté dans la famille élargie ou par des proches ${ }^{16}$. Trente six répondants citent la rue comme lieu de vie à cette période; $86 \%$ citent un seul lieu de résidence, $8 \%$ en citent $2,2 \%$ en citent 3 ou plus (non réponse : $4 \%$ ).

La durée moyenne de vie totale dans la rue est de cinq ans (écart-type 7 ans : les extrêmes vont de trois jours à 47 ans). Ainsi, $18 \%$ des personnes interrogées déclarent vivre dans la rue depuis plus de dix ans, $26 \%$ de trois à dix ans, $14 \%$ depuis un à trois ans, $13 \%$ de six mois à un an, et $29 \%$ depuis moins de six mois. L'âge moyen auquel on a connu la rue est de 29 ans (écart-type 11,8 ans : les extrêmes vont de 10 ans à 69 ans) : au total, $16 \%$ des répondants ont connu « la rue » avant l'âge de 18 ans.

La durée de présence est fortement liée à certains facteurs, qui sont, par ordre décroissant d'importance, l'âge auquel on a connu la rue pour la première fois (les personnes ayant connu la rue le plus tôt sont celles qui sont susceptibles d'y vivre le plus longtemps), le montant des dépenses journalières (les personnes qui ont les dépenses quotidiennes les plus faibles sont celles qui ont vécu le plus longtemps dans la rue), le sexe enfin (les raerae sont les personnes susceptibles de vivre le moins longtemps dans la rue, au contraire des hommes).

Les caractères spécifiques de cette population sont donc la prépondérance des hommes célibataires, et le poids important des personnes originaires des archipels éloignés. Mais il faut souligner la forte hétérogénéité des situations en matière d'âge et de durée de vie dans la rue, facteurs prépondérants pour expliquer les attitudes et comportements relevés plus loin.

\section{Un niveau de scolarisation et de formation préoc- cupant}

Le niveau scolaire relevé est très faible : près de la moitié $(44 \%)$ des personnes interrogées ${ }^{17}$ n'a pas le niveau « troisième », et $77 \%$ n'ont pas

14. Comparable à l'âge moyen de 34 ans obtenu dans l'enquête Pays de Loire, et aux résultats de l'INED à Paris avec $36 \%$ de 18-29 ans.

15. Données comparables aux $67 \%$ de célibataires et de $14 \%$ de couples dans l'enquête INED.

16. À rapprocher du résultat de l'enquête l'INED à Paris : un SDF sur quatre ne vivait à 16 ans ni avec son père, ni avec sa mère.

17. L'enquête quantitative a été menée par quatre enquêteurs, deux femmes et deux hommes répartis en binômes hommefemme. Trois des quatre personnes étaient bilingues français-tahitien ( $r e^{\prime} o$ mäohi). Le questionnaire a été conçu, testé et passé généralement en langue française, et une traduction en langue tahitienne de ce questionnaire avait été élaborée au préalable du terrain de l'enquête. Dans certains cas (mauvaise compréhension du français, enquêtés qui s'adressaient spontanément aux enquêteurs en langue tahitienne, explication de certains mots ou expressions...), les enquêteurs ont donc pu utiliser la traduction tahitienne. 
atteint le niveau baccalauréat ${ }^{18}, 16 \%$ ne savent pas ou ne veulent pas répondre. Parmi l'ensemble des répondants, l'âge moyen de fin de scolarisation est de 14 ans (écart-type : 2,8) : les deux tiers des répondants ont quitté l'école avant l'âge légal des 16 ans actuellement en application, compte tenu du fait que l'âge minimal légal était de 14 ans il y a quelques années encore en Polynésie française.

Parmi l'ensemble des répondants, seul un quart $(24 \%)$ déclare avoir reçu une formation professionnelle, et $5 \%$ ne savent pas. Les formations reçues sont de type "technique ", liées en majorité au bâtiment (42\%) à l'hôtellerierestauration-tourisme $(23 \%)$, à la mécanique $(10 \%)$ ou autre (artisanat, service aux personnes, agriculture-pêche...).

Les trois quarts $(72 \%)$ des personnes interrogées ont déjà travaillé, pendant une durée moyenne de sept ans et demi, mais avec des écarts très importants entre les individus (écarttype supérieur à la moyenne). Les emplois occupés concernent en majorité le bâtiment, le secteur secondaire en général ou le secteur primaire (agriculture, pêche), et sont des emplois peu qualifiés et précaires : on relève très peu de $\mathrm{CDI}$ et un travail non déclaré important ${ }^{19}$.

Les points communs à la population étudiés sont ainsi un niveau de scolarisation et de formation préoccupant, et des expériences générales d'emploi précaires et peu qualifiés, dont les effets se cumulent en se renforçant.

\section{Des conditions de vie socio-économiques précaires}

\section{Le quotidien des sans-abri tahitiens}

D’une façon générale, nonobstant les différences individuelles relevées au cours de la phase qualitative de l'enquête, le réveil est très matinal, entre $4 \mathrm{~h}$ et $5 \mathrm{~h}$ du matin, avant le début de l'activité de la ville : « après, il y a trop de bruit », « les gens commencent à arriver en ville ». Une rapide toilette est entreprise aux robinets d'eau publique du marché de Papeete (un petit groupe de sans-abri attend son ouverture tous les matins), aux toilettes publiques de la récente place Vaiete sur le front de mer, ou aux douches de la plage aux pirogues à Paofai.

Un rendez-vous quotidien en semaine est souvent évoqué : le petit-déjeuner offert à Vaininiore par le Centre Te Vaiete ${ }^{20}:$ " au moins, on n'a pas à se préoccuper du repas du matin », «j'y vais tous les jours, du lundi au vendredi », « on retrouve là-bas tous les copains ». Parmi les plus jeunes des personnes rencontrées, tous mineurs, on relève des évocations de petits-déjeuners offerts par les « copines » de la nuit (prostituées femmes ou raerae).

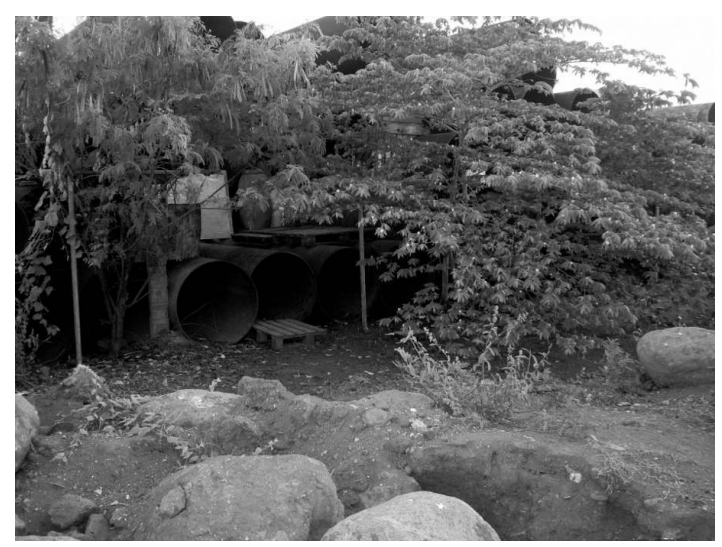

Рното 2. - Abri improvisé dans des tuyaux abandonnés sur un terrain vague (cliché de l'auteur)

La matinée est ensuite passée à discuter avec les «copains» dans des «spots» ou lieux de rencontre habituels, sur le domaine public de la commune de Papeete: l'espace de la rade dit «sable blanc », le pont de Motu Uta, la place Vaiete, etc. La matinée est ainsi remplie soit à se promener de «spot» en «spot», dans des parcours piétons apparemment inorganisés mais répondant à une géographie précise des différents «spots» de la ville et des activités possibles, soit à " travailler» pour pouvoir déjeuner. Les travaux cités sont toujours les mêmes : mettre en place les tables et chaises de quelques snacks de la ville en échange de nourriture, petits travaux évidemment non déclarés de jardinage ou de maçonnerie, chez des particuliers la plupart du temps.

18. À rapprocher des résultats de l'enquête INED : « $69 \%$ des SDF sont sortis de la scolarité avant la terminale, $5 \%$ n'ont jamais été scolarisés, 41 \% sont sans diplôme (pas de différences hommes / femmes), 17 \% ont un diplôme supérieur ou égal au bac ». La situation apparaît donc comme encore plus préoccupante dans notre enquête.

19. Les résultats sont très similaires dans l'enquête Pays-de-Loire : 7 personnes sur 10 n'ont jamais eu d'emploi ou ont exercé des activités professionnelles précaires, alors que ce taux est plus élevé dans l'enquête INSEE de $2001: 90 \%$ ont travaillé au cours de leur vie, huit fois sur dix comme employés ou ouvriers (Rochère, $2003: 1$ ).

20. Le centre Te Vaiete est une structure associative créée par le Secours catholique polynésien, déclarée à la direction des Affaires sociales comme « gîte officiel » pour fournir des attestations de résidence. Depuis 1995, des bénévoles servent des repas complets (plat chaud, dessert, barres de chocolat, jus de fruits) le matin de $6 \mathrm{~h} 30$ à $8 \mathrm{~h}$ environ du lundi au vendredi. Les produits (en format collectivité) sont donnés par des importateurs et des commerçants. Le local, adjacent à celui du Club de prévention spécialisée, a été mis à disposition par la commune de Papeete, qui prend également en charge les frais d'électricité et d'eau. Le local dispose également d'une douche, d'une machine à laver le linge et d'un sèche-linge. 
Le déjeuner est léger et les mêmes réponses apparaissent, quand déjeuner il y a : « un cassecroûte » (le "sandwich» à la française), «du pain et une boîte de pâté », consommés debout en général. Le programme est le même l'aprèsmidi, balades de «spot» en "spot», ou petits travaux de fin d'après-midi pour pouvoir dîner. Les travaux cités consistent là-encore à débarrasser les tables et chaises des snacks et à « nettoyer » (balayer, passer un jet d'eau), en échange de nourriture, à vendre à la sauvette des fruits glanés ou cueillis dans les rares jardins des alentours, etc.

Le dîner est en général plus consistant que le déjeuner : « du riz avec de la sauce » (acheté aux roulottes de la place Vaiete), "du pain et une boîte de maquerelle » (ou de sardine à la tomate, achetés en commerce) consommés soit debout soit assis dans un « spot tranquille».

Les repas quotidiens cités qui font l'objet d'un achat reviennent à 200 FCFP (environ $1,7 €$ ), chiffre cité en grande majorité : "51 FCFP la boîte, soit 102 FCFP les deux boîtes, et deux pains [baguettes] à 47 FCFP ». Le plat acheté aux roulottes fait l'objet d'une remise par le commerçant, car il ne contient ni viande ni poisson, et est même parfois offert.

Une fois pris le repas du soir, la soirée est passée à attendre le moment de dormir, toujours tardif pour laisser passer l'activité nocturne de Papeete (boîtes de nuit, jeunes des quartiers de l'agglomération qui "traînent en ville et en bande »...), et éviter ainsi les problèmes consécutifs à l'ingestion d'alcool des noctambules : « je ne me couche pas avant $23 \mathrm{~h} »$, « je vais dormir vers minuit - une heure du matin ».

De fait, les nuits sont courtes, d'une durée moyenne de cinq heures, et agitées par la présence indésirable des "jeunes des quartiers" rôdant en ville, ou des rondes des policiers nationaux de la direction de la sécurité publique (équivalent de la BAC métropolitaine) appelés les « ninja » du fait de leur uniforme entièrement noir. La nuit constitue ainsi pour ceux que l'opinion publique juge " dangereux » le moment de tous les dangers : agressions par d'autres sansabri (peu cité) ou par «des voyous des quartiers » (plus généralement cité), vols de vêtements ou d'argent pendant le sommeil, et inconfort et froid (en saison sèche) des nuits passées par terre sur un carton (to'eto'e roa). La nuit constitue aussi le moment de l'argent facile pour les mineurs rencontrés et, notamment, celui de la prostitution masculine des vendredis et samedis soirs.
Nourriture insuffisante en qualité et en volume, durée forcément réduite d'une nuit agitée, inconfort des conditions de sommeil, absence générale de couvertures et de vêtements « longs »(pantalons, tricots à manches longues), il ne semble dès lors pas étonnant que certaines personnes avouent souffrir du froid, même sous un climat plus clément que celui de la France métropolitaine, à l'encontre des lieux communs généralement formulés ( la misère est moins dure au soleil »...).

Les conditions de vie des personnes rencontrées paraissent ainsi particulièrement précaires, notamment en matière de besoins élémentaires. Le toit est constitué par un endroit abrité de la pluie, et des regards des passants si possible, et retiré des principales artères automobiles, avec un carton (« je vérifie avant s'il est propre et je le sens ») pour matelas. Le linge est en général fourni par la Croix rouge ou les Églises ; on est habillé d'un short, d'un tee-shirt et d'une paire de savates, et le minimum gardé avec soi dans un sac à dos : un short et un ou deux « tricots» de rechange, rarement un pantalon et une chemise à manches longues ( « le pyjama ») pour dormir, et une carte nationale d'identité quand on en a une. Le linge est lavé aux fontaines publiques de la ville (place Vaiete), ou grâce aux machines à laver que rend disponibles le Centre de jour ( " mais ça fait honte devant tout le monde »). Les transports se font bien sûr à pied, le prix minimum d'un truck correspondant à la valeur monétaire d'un repas. Les loisirs sont limités à la discussion avec les "copains", à regarder les voitures et les gens passer, ou encore à boire et/ou fumer en groupe. Le terrain de sport dont dispose le Centre de jour est peu utilisé, hormis quand un animateur ou un stagiaire du Centre décide d'organiser une rencontre amicale.

\section{Des relations sociales entre " pairs 》}

Contrairement aux idées répandues d'une désocialisation et d'une rupture familiales totales des sans-abri, des contacts sont gardés avec la famille : $42 \%$ des répondants déclarent avoir gardé des liens avec un membre au moins de leur famille. Mais ces liens sont en très grande majorité occasionnels (seuls $3 \%$ d'entre eux se voient « souvent ») et privilégient les parents dans $50 \%$ des cas (dont 15\% la mère seule, 15\% la "famille nucléaire », $14 \%$ les deux parents et $5 \%$ le père seul), les frères ou les sœurs dans $22 \%$ des cas $^{21}$, les cousins et cousines dans $12 \%$ des cas, les enfants $(6 \%)$, les grands-parents $(5 \%)$,

21. Les résultats de l'enquête INED menée à Paris donnent des résultats comparables : $29 \%$ des sans-abri interrogés ont eu un contact avec leur père durant l'année écoulée, et $37 \%$ avec leur mère. 
les oncles et les tantes ( $3 \%$ ), les concubins ou les copains ( $2 \%)$. La phase qualitative de l'enquête a en outre permis de mettre en évidence des contacts parfois entretenus avec des commerçants du quartier dans lequel les sans-abri passent leurs nuits, qui leur fournissent de la nourriture (jamais d'argent) sans contrepartie, ou contre de menus travaux.

En matière de relations sociales, les personnes rencontrées ont donc peu de contacts avec d'autres personnes, hormis avec celles vivant dans la rue: "les copains". Elles mettent en grande majorité l'accent sur la stigmatisation dont elles font l'objet au quotidien : « les gens nous regardent de côté », " quand je demande un cent franc, on me dit que je ferais mieux d'aller travailler ». Comme l'ont noté E. Goffman (1975) et S. Paugam (1991) notamment, les phénomènes d'exclusion et de déqualification sociales sont le produit d'une construction sociale, à laquelle les personnes soumises à la stigmatisation adhèrent en intériorisant certaines représentations négatives ( « Ça fait honte »).

Par ailleurs, elles ont également peu de contacts avec les institutions. Seules quelques citations spontanées ont été relevées, et ne concernent que de rares structures, celles qui sont le plus souvent et en contact direct avec eux : le Club de prévention spécialisée (« le Centre de jour ») qui n'est jamais cité en tant que tel, mais au travers des prénoms de ses animateurs de rue, le centre Te Vaiete jamais cité non plus en tant que tel mais sous la figure charismatique de son responsable et animateur, les repas gratuits le premier jeudi du mois et les structures qui les organisent («les Églises»). Nous n'avons pas noté de mentions spontanées des centres d'hébergement, qui n'apparaissent qu'en relançant l'entretien sur ces derniers. De la même façon, nous n'avons relevé aucune citation spontanée concernant les institutions chargées d'œuvrer auprès de ce public (direction des Affaires sociales, Caisse de prévoyance sociale, Office polynésien de l'Habitat...). En relance, il apparaît auprès des personnes rencontrées une perception négative de ces institutions : les principaux griefs concernent l'absence ou le peu de rencontres, l'absence de suivi des dossiers administratifs en cours, des jugements moralisateurs sur la situation de la personne plutôt qu'une écoute objective ou une réelle aide : « Les obstacles à leur réinsertion sont d'ordre psychologique et social : il faut qu'ils acceptent de se réinsérer dans la société, de s'intégrer, d'être un peu comme tout le monde, et je ne sais pas s'ils veulent devenir comme tout le monde s'ils sont SDF » (un travailleur social).
À la question « quelles sont les personnes ou structures qui aident les gens qui vivent dans la rue ? », et malgré un taux de non-réponse important, seules deux structures sont citées spontanément par les répondants : le centre Te Vaiete (59\% des répondants), et le Centre de jour $(51 \%)$, qui se trouvent être les structures en contact au quotidien avec les sans-abri.

Des acteurs institutionnels tels que les « travailleurs sociaux » (constitués des intervenants de la direction des Affaires sociales) et la « Caisse de prévoyance sociale » recueillent entre un quart et un tiers des réponses, autant que la structure d'hébergement pour hommes le Bon Samaritain. La réponse « autres » regroupe des réponses minoritaires telles que « la Justice» (4 réponses, soit $1 \%$ du total des répondants), « les clients » pour les prostituées (4 réponses, soit $1 \%$ du total), « les commerçants » (3 réponses, soit $1 \%$ ), et pour moins de $1 \%$ des réponses au total les voisins (1 réponse), la Croix-Rouge (1 réponse), la ccism (1 réponse), la commune (1 réponse).

D’une façon générale, on peut noter que les structures privées et associatives recueillent plus de réponses spontanées que les structures institutionnelles et l'absence remarquable des communes qui ne recueillent qu'une seule réponse spontanée.

Devant cette absence quasi générale de contacts humains, personnels comme institutionnels, et la rupture familiale constituant en premier lieu le statut d'errant (Moreau de Bellaing et Guillou, 1995 : 184), il apparaît chez les personnes vivant dans la rue une nécessité : se constituer en " groupe de copains », tous vivant dans la rue, partageant les mêmes expériences et subissant le même regard de la part de la population et des institutions. Nous avons noté de façon quasi générale une mise en commun des ressources au sein des groupes ainsi constitués, notamment en matière alimentaire, ou de consommation d'alcool et de cannabis pour partager un " trip ». Lorsque l'un des membres du groupe dispose de liquidités (perception mensuelle d'une petite pension, rémunération de petits « boulots », argent issu de trafics divers...), il en garde une partie pour lui ( je cache mon argent dans mon slip pour ne pas me le faire voler pendant la nuit »), souvent la moitié de la somme, et l'autre moitié est versée au « pot commun » pour l'achat et la consommation en général immédiate de nourriture ou parfois de psychotropes.

Il semble se recréer ainsi une "communauté de destin " se détachant peu à peu d'une société dont ils s'estiment rejetés ou mis à l'écart, 
renforçant par là leur statut de « marginaux » ou de « déviants » : c'est l'interaction, le regard des autres porté sur quelqu'un qui fait de lui un déviant (Becker, 1985). Le regard et le jugement d'autrui ont bien une fonction de désignation, type de relation engagée entre l'attribut (le stigmate) et le stéréotype (Goffman, 1975). La seule solution pour le sans-abri tahitien est d'adopter une position "d'évitement», en refusant de «faire la manche » pour se nourrir, en prenant soin de ne pas paraître négligé, en portant toujours des vêtements propres et d'apparence correcte (ni déchirés ni troués), et en évitant de stationner trop longtemps au même endroit pour éviter de se faire repérer non tant par les forces de police que par d'éventuelles connaissances, phénomène plus fréquent et donc sans doute plus redouté dans une ville de la taille de Papeete que dans une grande ville française. De la même façon, les seuls centres d'hébergement existant aujourd'hui à Tahiti sont pris en charge par des associations religieuses, notamment catholiques. Les discours qui y sont tenus par les responsables et travailleurs sociaux sont perçus comme normatifs et moralisateurs par les personnes hébergées ("il faut supporter à chaque fois leur prêchi-prêcha, une prise de tête »), renforçant leur sentiment de culpabilité vis-à-vis de leur situation, et les horaires et la discipline jugés trop stricts (" c'est pire que la prison », « c'est comme à l'armée ») par des personnes qui vivent parfois dans la rue depuis de longues années.

\section{Les inconvénients perçus à vivre dans la rue}

Les problèmes rencontrés lorsqu'on vit dans la rue sont nombreux selon les personnes interrogées. Parmi celles qui trouvent un inconvénient à vivre dans la rue (seuls $14 \%$ n'en trouvent aucun), en moyenne 2,6 inconvénients sont donnés.

Contrairement à l'image communément partagée du sans-abri représentant un danger public pour les citoyens "normaux ", la rue est vécue par les personnes qui y vivent comme un lieu de danger, d'agressions et de vols (62\%), et notamment par les plus jeunes et les plus âgés d'entre eux, et par les femmes. Les agressions dont font l'objet les personnes vivant dans la rue semblent de plus en plus nombreuses depuis quelques années, et fragilisent encore plus les personnes qui y vivent, qu'il s'agisse des plus jeunes, des plus âgés, ou des femmes ( je suis tout le temps agressé et tabassé par les plus forts », « quand on est vieux, on nous bouscule, on nous vole et on nous tabasse souvent », " quand tu es une femme et que tu vis dans la rue, tu as intérêt à te trouver un tane [compagnon] pour te protéger $»)$.

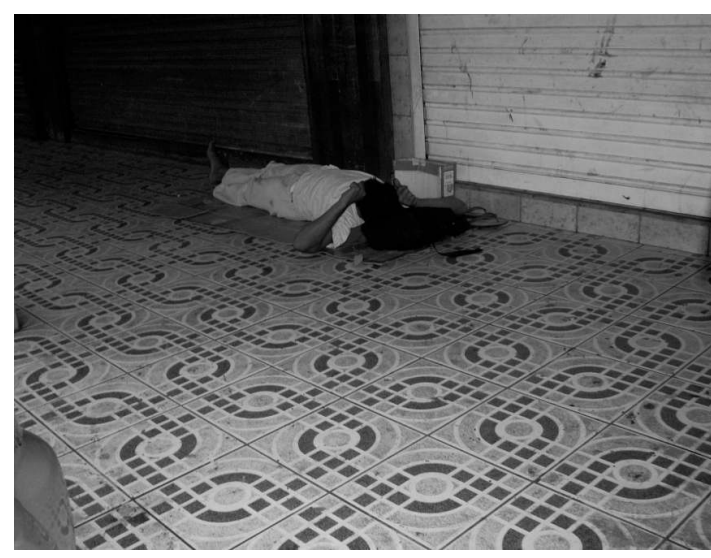

Рното 3. - Un carton d'emballage comme couche devant le rideau de fer d'un commerce où un sans-abri passe la nuit (cliché de l'auteur)

Le fait de « ne pas avoir d'endroit pour dormir » (31\% des répondants) constitue bien sûr la spécificité des personnes vivant dans la rue (« quand il fait mauvais temps, c'est trop dur de dormir dehors, ce n'est pas confortable ») et le deuxième inconvénient cité en moyenne. Mais cette dimension recouvre également la concentration croissante de ces personnes à Papeete, et le fait que « d'anciens » sans-abri doivent laisser place à de plus récents, ou encore que les plus jeunes d'entre eux, et notamment les mineurs, doivent trouver des lieux pas encore territorialisés par leurs prédécesseurs ( $«$ je dors là où il y a de la place »). La dispersion des lieux de vie et leur caractère aléatoire et changeant illustrent ce phénomène: les sans-abri polynésiens ne se concentrent plus seulement autour du marché de Papeete, zone centrale et historique et point de ralliement, mais investissent désormais des quartiers éloignés du centre-ville et de ses commodités : Fare Ute, Motu Uta, Taunoa... dormant à même le sol sur un carton, ou encore dans un immeuble en construction, au milieu des matériaux de construction sur un chantier, dans un endroit abrité au milieu d'un terrain vague, dans des voitures aux portes laissées ouvertes par leurs propriétaires, etc., formes d'« appropriation» occasionnelle et sans cesse remise en question.

La faim, troisième réponse énoncée par près d'un quart des personnes interrogées (« depuis que je vis dans la rue j'ai tout le temps faim », « la vie est dure quand on ne sait pas comment trouver à manger »), est de nouveau un coup porté au lieu commun souvent entendu, y compris de la part d'élus municipaux : «en Polynésie, on ne peut pas avoir faim ». L'argument de fruits et de poissons librement disponibles ne résiste en effet pas à l'analyse, la concentration 
urbaine à Papeete ayant fait disparaître nombre d'arbres fruitiers, et rendu délicat l'accès au lagon. Selon Serge Paugam (1991 : 219) :

« la disqualification est avant tout une épreuve, non pas seulement en raison de la faiblesse des revenus ou de l'absence de certains biens matériels, mais surtout en raison de la dégradation morale que représente dans l'existence humaine l'obligation de recourir à l'appui de ses semblables et des services d'action sociale pour obtenir de quoi vivre dans des conditions décentes. »

Les réponses « le regard des autres » $(20 \%)$ et « la saleté » $(17 \%)$ (« ce que je n'aime pas, c'est que je sens mauvais tous les jours, et j'ai honte... », " même les chiens sont mieux traités que nous : on leur donne à manger tous les jours sans leur demander de travailler... », " on nous regarde méchamment», "ce que je voudrais avant tout, c'est de ne pas nous regarder comme le font les gens : nous ne sommes pas des monstres ») sont souvent complémentaires et mettent l'accent sur la stigmatisation forte dont font l'objet les sans-abri, les renforçant encore plus dans leur statut de marginaux et de " déviants", y compris de la part de personnes dont le premier discours spontané évoque la liberté de la vie dans la rue et le sentiment de maîtriser leur propre corps.

D'autres réponses apparaissent de façon plus minoritaire, et notamment l'ennui éprouvé dans la rue $(15 \%)$ du fait de l'absence d'activités, et le fait d'être séparé de ses enfants $(10 \%)$. L'absence d'hygiène quotidienne, et un suivi de santé incertain ou insuffisant malgré la gratuité des soins mise en place pour les populations les plus défavorisées, sont évoqués soit par les plus jeunes, soit par les personnes frappées de pathologies invalidantes (goutte, handicap physique...) ou nécessitant un suivi médical régulier (diabète, maladies cardio-vasculaires, problèmes dentaires graves, cancers... $)^{22}$. L'hygiène quotidienne est bien souvent limitée à la douche publique et à des ablutions aux robinets publics. Tous évoquent le peu d'infrastructures en la matière à
Papeete. La contraception semble inexistante ou très irrégulière.

\section{Des dépenses quotidiennes variables}

Il s'avère donc que les conditions de vie sont particulièrement précaires pour la population étudiée : $38 \%$ des répondants déclarent dépenser 500 FCFP par jour ou moins (soit environ $4,2 €$ ), soit un maximum de 15000 FCFP par mois $(125 €)$. Ces dépenses recouvrent principalement la nourriture, mais également des besoins annexes (boissons, tabac à rouler). L'alimentation est bien le poste budgétaire pour lequel les écarts sont les plus importants entre les groupes de revenus, et encore plus quand on s'attache aux populations très défavorisées (Cavaillet et Momic, 2004) : le poids des achats alimentaires dépasse $50 \%$ des dépenses quotidiennes pour près de la moitié des personnes interrogées, alors que ce taux n'est que de $19 \%$ en moyenne pour la population totale dans la zone urbaine de Tahiti.

Selon l'ISPF (2005), le seuil de pauvreté absolue correspond à un revenu annuel inférieur à 57796 FCFP (environ $480 €$ ) et concernerait moins de $0,5 \%$ de l'ensemble des familles polynésiennes. Ce seuil (environ 160 FCFP par jour, soit un peu plus d'un euro) concerne $28 \%$ des sans-abri interrogés. Toujours selon les mêmes données de l'ISPF, le seuil de pauvreté relative est de 51470 FCFP (environ $430 €$ ) par mois et par unité de consommation, et toucherait 18,6\% des ménages polynésiens ${ }^{23}$. Ce seuil (1 700 FCFP par jour soit environ $14 €$ ) concerne $76 \%$ des personnes interrogées vivant dans la rue.

Cumulant un niveau de scolarisation et de formation préoccupant, une précarité économique et une rupture du lien social primaire, les sans-abri tahitiens font donc face à un phénomène de désaffiliation au sens où l'entend R. Castel (1991), auquel chaque type de sansabri, sinon chaque individu, s'adapte peu ou prou en fonction de stratégies individuelles. La notion de désaffiliation paraît plus intéressante à

22. Les problèmes de santé les plus fréquents relevés par les Services de Santé Publique chez les sans-abri dans l'agglomération de Papeete, sans pour autant identifier cette population en tant que telle dans les statistiques de fréquentation et de types de soin apportés, sont de graves infections cutanées (prodermite, infections purulentes...) qui constituent selon les structures entre 30 à $50 \%$ des problèmes constatés et qui nécessitent à la fois des traitements en urgence et un suivi régulier, des problèmes dentaires (avec des complications sous forme de septicémies et de problèmes cardiaques), le diabète et la goutte qui nécessitent des traitements quotidiens, des insuffisances respiratoires (qui entrainent des bronchites chroniques et parfois même des emphysèmes pulmonaires), toutes pathologies qui nécessitent un suivi médical, selon deux grandes modalités : suivi médical régulier et quasi quotidien (diabète, goutte, problèmes respiratoires...) ou plus ponctuel, au mois (problèmes cutanés...).

23. Ces taux sont sans doute très nettement sous-estimés puisque l'IsPF considère le seuil de pauvreté relative en dessous de $50 \%$ du niveau de vie médian, alors que l'Europe utilise le seuil de $60 \%$ du niveau de vie médian. De plus, l'ispF prend en compte dans ce niveau de vie les ressources non monétaires (autoconsommation, échanges...) qui constituent en moyenne 6 à $7 \%$ des ressources totales d'un ménage moyen polynésien, alors que l'INSEE en France ne prend en compte que le revenu disponible, c'est-à-dire les revenus financiers y compris les prestations sociales, nets des impôts directs. 
utiliser que le concept d'exclusion, puisque l'accent est mis sur le processus de rupture du lien social, sur une dynamique plus que sur un état. L'existence de "zones de cohésion sociale », reposant sur l'association d'un niveau d'emploi et d'une densité de réseau relationnel, conduit ainsi à définir un processus de vulnérabilité progressive en cas de ruptures, qui déboucherait sur une situation de désaffiliation.

Par ailleurs, l'utilisation du terme " exclusion » conduit, au moins localement, à considérer ensemble des populations très diverses et aux trajectoires de vie très différenciées : jeunes des quartiers sociaux ou placés dans des foyers d'action éducative, sans emploi, toxicomanes, anciens détenus en liberté surveillée, personnes handicapées ou âgées, sans aucune référence spécifique aux sans-abri. Ainsi la délibération 94-148 AT du 08/12/94 instituant un Fonds d'action sociale (FAS) au Régime de solidarité territoriale (RST) indique que ce Fonds est destiné à « [...] venir en aide aux catégories défavorisées, notamment les handicapés, les personnes âgées, les enfants en danger, les familles ou personnes en détresse ». La délibération 95-135 AT du 24/08/95 modifiant la précédente précise que :

«[...] les programmes d'action sociale du régime de solidarité territoriale comprennent : l'éducation familiale et sociale ; la prévention de la maltraitance et de la délinquance des mineurs; les programmes destinés aux personnes âgées ; les actions en faveur du handicap médical ou social ; le développement de l'action sociale dans les archipels; les mesures en faveur du traitement de l'exclusion sociale. »"

De même, la délibération 2001-157 APF du 06/09/01 relative aux associations pour l'insertion indique que les missions de ces associations consistent à :

«[...] accueillir prioritairement les personnes en situation ou en risque de marginalisation, les personnes relevant de la liberté surveillée, les mineurs de seize ans en rupture familiale et les personnes venant d'achever une période d'incarcération ou de désintoxication $[\ldots]$ »

Cette absence de référence formalisée aux personnes vivant dans la rue se retrouve dans les documents de travail des différents acteurs institutionnels. Ainsi, la fiche-type d'un budget du Régime de solidarité de Polynésie française (RSPF) fait apparaître les différents programmes d'action sociale en fonction des axes suivants : « insertion des familles / insertion des jeunes / insertion des personnes âgées / insertion des personnes handicapées / actions de formation ».
Les sans-abri polynésiens sont donc en quelque sorte "noyés » dans les textes réglementaires avec d'autres types de population, sans être identifiés en tant que tels, et assimilés sinon réduits par les acteurs de terrain selon les situations à un " jeune toxicomane », une " personne âgée » en difficulté économique, un «malade mental» errant dans la rue, un «ancien détenu ", un couple "chômeur» avec des enfants, etc. sans jamais, ou rarement, prendre en compte la globalité, certes complexe, de leur situation.

L'analyse quantitative "moyennise » également des situations individuelles très différentes pour établir le portrait-type d'un «sans-abri » auquel aucune personne rencontrée sur le terrain ne correspond vraiment. Ainsi, la dépense moyenne quotidienne déclarée est de 1470 FCFP (donc inférieure au seuil de pauvreté relative), mais elle connaît un écart-type très important dû aux différences de situation : les extrêmes vont de 0 à 10000 FCFP. Il existe de nombreuses différences entre les personnes interrogées en fonction de leur profil :

- les femmes sont sur-représentées à la fois parmi les personnes déclarant ne rien dépenser par jour (zéro FCFP) et parmi celles déclarant dépenser plus de 4000 FCFP par jour du fait du poids des prostituées parmi ces dernières réponses,

- les raerae sont sur-représentés parmi les personnes déclarant dépenser plus de 1000 FCFP par jour, et surtout parmi celles déclarant dépenser plus de 4000 FCFP par jour (influence également de la prostitution parmi cette population),

- la probabilité de dépenser plus par jour est d'autant plus forte que la personne vivant dans la rue est plus jeune : $22 \%$ des moins de 40 ans déclarent dépenser 2000 FCFP par jour ou plus (influence des dépenses en pakalolo ou cannabis), contre seulement $10 \%$ des 40 ans et plus.

La prostitution, le trafic de pakalolo et les vols sont des facteurs favorisant les dépenses élevées, sans que celles-ci soient absolument liées aux premières.

Les personnes interrogées déclarent près de deux $(1,6)$ moyens de se procurer des ressources.

« Faire charité » est la principale ressource pour plus d'un sans-abri sur deux (56\%). Une personne vivant dans la rue interrogée sur cinq $(19,6 \%)$ déclare recourir à des «petits bou- 
lots $»^{24}$ (《j'aide à décharger les bateaux. Tous les travaux qu'on me propose, je fais $»)$ : petits travaux de maçonnerie pour des particuliers $(7 \%)$, aide aux snacks et vendeurs du marché de Papeete $(3 \%)$, entretien des jardins de particuliers $(2 \%)$, pêche $(2 \%)$, ménage et gardiennage chez des particuliers $(2 \%)$, vente de fruits donnés ou volés $(2 \%)$ et autres (revente des bouteilles de bière vides, gardiennage de voitures, couture... $3 \%$ ).

Mais des façons annexes ou complémentaires de gagner de l'argent ou de se procurer de la nourriture existent. Un sans-abri sur trois déclare des activités illicites : $20 \%$ avouent voler (dans les voitures, à l'étal des magasins, les passants ivres...), $9 \%$ recourir à la prostitution, $2 \%$ procéder au trafic de pakalolo (cannabis) et $1 \%$ protéger des prostituées. D'autres moyens de subvenir à ses besoins sont également déclarés de façon minoritaire : argent donné par la famille $(8 \%)$, économies $(2 \%)$, emploi régulier $(3 \%)^{25}$. En Polynésie française, comme le note Serge Paugam (1991) pour la France, « le travail ne protège plus de l'extrême pauvreté. Il ne suffit pas d'avoir un emploi pour échapper à la précarité ».

Parmi les personnes rencontrées, $15 \%$ déclarent percevoir une pension régulière (d'un montant de 40000 FCFP en moyenne soit $330 €$ environ) : retraite $(8 \%)$, pension invalidité $(2 \%)$, pension adulte handicapé $(2 \%)$, prestations familiales $(1 \%)$. Mais il ne semble pas que la personne en soit toujours la bénéficiaire : elle peut être frappée d'incapacité juridique et avoir été placée sous tutelle, les fonds peuvent être prélevés directement par la famille (sœur, parent...) ou réservés aux enfants comme nous l'avons relevé au cours de l'enquête.

\section{L'alimentation des sans-abri à Tahiti}

\section{Une alimentation au quotidien insuffisante en quantité et en qualité}

Hormis l'idée que les personnes vivant dans la rue l'étaient de leur plein gré, par volonté délibérée de ne pas vivre au sein du domicile familial (« ils sont dans la rue parce qu'ils le veulent bien ", " tout le monde a de la famille en Polynésie, donc personne n'est sans abri » etc.), dans un pays où les relations sociales et surtout familiales sont censées être restées traditionnelles et denses, un des lieux communs les plus souvent énoncés à propos des sans-abri dans l'agglomération de
Papeete est qu'ils n'ont pas à souffrir de la faim puisque végétaux et poissons sont librement disponibles. Outre que cette affirmation («il suffit de tendre le bras pour cueillir des fruits disponibles toute l'année »), partagée par certains des élus municipaux et acteurs institutionnels « de proximité » rencontrés, est issue d'une perception mythique d'un Éden polynésien où la nourriture est toujours et librement disponible en abondance (Serra Mallol 2005) ou d'une forme inversée "d'idéologie du retour» qui prend la forme d'une injonction d'un retour aux zones rurales où l'accessibilité aux ressources naturelles est plus aisée, elle ne reflète en rien la réalité dans l'agglomération urbaine tahitienne, et surtout à Papeete même où sont concentrées $80 \%$ des personnes vivant dans la rue : jardins privés clôturés, passage de plantations vivrières au profit des fleurs dans les jardins, disparition avérée depuis plus d'une cinquantaine d'années des plantations vivrières communautaires en lisière des communes ou en fond de vallée au profit de rares plantations commerciales à Tahiti... De la même façon, la fréquentation et la pêche accrues des lagons de l'agglomération, et du chef-lieu en particulier, font qu'il est désormais nécessaire de disposer d'un matériel minimal (canne à pêche, filet, sinon bateau ou pirogue) pour pouvoir assurer sa subsistance en matière de produits de la mer. Sans compter la nécessaire préparation culinaire de ces produits qui impose le recours à un appareil de cuisson et aux ustensiles de cuisine, dont ne disposent évidemment pas la quasitotalité des sans-abri rencontrés.

De fait, la réalité est bien différente. Le seul repas complet pour beaucoup des personnes rencontrées vivant dans la rue est celui du matin, grâce à la distribution gratuite de repas complets organisée bénévolement en semaine par un responsable religieux : $38 \%$ des personnes interrogées déclarent bénéficier de façon plus ou moins régulière de ce service. Les autres se satisfont d'une nourriture frugale («café-pain-beurre» en majorité, pain accompagné d'une boîte de sardines ou de pâté, sandwich, pain seul, restes de nourriture glanés dans des poubelles, café sucré seul) ou même de rien. Hormis le repas offert par le Secours catholique polynésien, les autres achètent leur repas du matin (37\%), ou se le voient offrir (12\%) par charité ou par échange de services, et $5 \%$ le partagent avec d'autres personnes de « la rue » (sans-abri ou prostituée). Six pour cent déclarent avoir mangé le matin dans un centre d'hébergement et $2 \%$ dans leur famille ou chez un ami. 
À propos du repas de milieu de journée, $46 \%$ déclarent ne rien manger ${ }^{26}$. Les autres prennent les mêmes en-cas que ceux cités pour le matin ( « casse-croûte », pain accompagné d'une boîte de conserve, restes de nourriture...). Seuls $13 \%$ prennent un plat chaud ou un repas complet, acheté ou offert. Parmi ceux qui déclarent manger à midi, $54 \%$ achètent leur nourriture, $19 \%$ se la voient offrir par charité ou par échange de services, $7 \%$ la partagent avec d'autres sans-abri ou des prostituées, $7 \%$ déclarent la voler, $2 \%$ utilisent des restes du repas gratuit du matin, $6 \%$ déclarent avoir mangé à midi dans un centre d'hébergement, et $5 \%$ dans leur famille ou chez des amis.

Le repas du soir est un peu plus fréquent que celui du midi : seuls $10 \%$ déclarent ne rien manger. Il est plutôt composé d'un plat chaud (45\%) acheté dans une roulotte ${ }^{27}$ ou encore partagé avec d'autres sans-abri, de pain accompagné d'une boîte de sardines ou de pâté (22\%) achetés dans le commerce ou encore partagés, de restes de nourriture trouvés dans les poubelles $(7 \%)$, de café seul ou accompagné de pain beurre $(6 \%)$, de pain seul $(3 \%)$ ou de fruits seulement ( $2 \%$ ). Seuls $3 \%$ bénéficient d'un repas complet le soir, offert en général par leurs clients aux personnes vivant dans la rue et qui se prostituent ; $5 \%$ déclarent avoir mangé le soir au domicile de la famille ou chez des amis, et $3 \%$ dans un centre d'hébergement.

On notera l'absence de dispositif institutionnel d'alimentation pour les sans-abri : même les bons alimentaires distribués avec parcimonie par les Services Sociaux territoriaux ne sont pas vraiment adaptés, constituant des bons d'achat dans certains commerces pour des produits qui nécessitent ensuite une préparation ou une cuisson dont les ustensiles font souvent défaut aux personnes vivant dans la rue.

\section{L'importance de la nourriture}

Nous avons montré ailleurs l'importance tant qualitative que quantitative de la nourriture et des prestations alimentaires dans la culture polynésienne, dont la principale caractéristique est d'être basée sur une notion d'abondance quantitative et sur l'irrégularité des prises (Serra Mallol, 2007). Si cette irrégularité des consomma- tions est avérée chez les sans-abri interrogés, il s'agit ici bien plus souvent d'une contrainte de fait que d'habitudes de consommation. De même, la notion d'abondance est exclue des discours. Le caractère compensateur, principe de plaisir plus physique qu'organoleptique (l'expression tahitienne $p a$ 'i $a$, s'éprouver à la fois rassasié et empli de nourriture) que nous avions relevé, est ici absent (ou en creux, comme souhait : « bien manger, c'est être rassasié, pour faire la sieste après ») : on mange d'abord par nécessité vitale, « je mange pour survivre », « manger ce qu'il faut pour avoir des forces », « je mange pour être en forme», «si je ne mange pas je risque d'avoir des problèmes de santé », etc. Le pain est omniprésent, ainsi que les conserves bas de gamme de bœuf en boîte (punu pua'atoro), de poisson (sardines, maquereau) ou de légumes (lentilles, haricots). La viande est rarement évoquée (et plutôt le poulet que les viandes dites rouges), sauf comme aliment considéré comme " important ", au même titre que le poisson.

À la question "pour vous, bien manger, qu'est-ce que cela signifie? », nous relevions, auprès de personnes issues de ménages certes modestes mais « domiciliées », des réponses liées au caractère culturel de la nourriture, qui évoquaient les aliments perçus comme «typiques », « traditionnels », de l'alimentation tahitienne: fruits et tubercules locaux (taro, fề' $i$, 'uru...), poisson cuit ou cru préparé «à la tahitienne » (légèrement macéré dans de l'eau de mer ou du citron, puis mélangé à du lait de coco), préparation à base de sauce au lait de coco (poisson cru mais également porcelet au lait de coco, fäfaru, po'e, etc.). Dans les réponses données par les personnes vivant dans la rue, rien de tout cela. L'objectif rarement atteint est d'être rassasié, d'atteindre cette plénitude physique et ce bienêtre que procure un estomac bien rempli.

\section{Une consommation répandue d'alcool et de can- nabis.}

Contrairement au lieu commun, les personnes vivant dans la rue ne sont pas toutes fortes consommatrices d'alcool. Toutefois, la consommation d'alcool est relativement élevée parmi cette population, puisque seuls $15 \%$ déclarent ne jamais boire ou occasionnellement (moins

26. À rapprocher des résultats de l'enquête INED à Paris : « $59 \%$ des hommes et $78 \%$ des femmes mangent tous les jours à midi (souvent un sandwich) ; $16 \%$ des hommes et $10 \%$ des femmes n'ont jamais pris de repas à midi au cours de la semaine précédant l'enquête" " (Firdion et Marpsat, 2000).

27. Les roulottes sont des véhicules de type camionnettes, à l'arrière desquels est aménagée une cuisine servie par les côtés Ambulants, leur activité est principalement nocturne. Près de trois cents roulottes sont installées en Polynésie française, dont 128 pour la seule île de Tahiti. Le client peut consommer un plat populaire et bon marché sur place, attablé devant un panneau amovible installé sur les côtés du véhicule et assis sur un tabouret amené par le « roulottier », ou dans quelques cas autour de tables en plastique, ou acheter des plats à emporter pour consommation à domicile. 
d'une fois par semaine), beaucoup citant un abandon de la consommation d'alcool au cours de l'année après signature d'un contrat moral avec une Église du type "Croix bleue ${ }^{28}$ ». Les autres personnes interrogées se partagent entre $36 \%$ qui déclarent boire une fois par semaine, $32 \%$ déclarant boire une fois par jour, et $13 \%$ plusieurs fois par jour, cette dernière fréquence de consommation pouvant atteindre en volume jusqu'à plusieurs litres de vin bas de gamme pour les plus anciens des sans-abri pour lesquels les dépenses quotidiennes en alcool dépassent celles consacrées à la nourriture.

La consommation de pakalolo (cannabis local) est plus hétérogène puisque $35 \%$ déclarent ne jamais en fumer ou occasionnellement (moins d'une fois par semaine). En revanche, $11 \%$ déclarent en fumer une fois par semaine, $27 \%$ une fois par jour et $22 \%$ plusieurs fois par jour ; ce dernier type de consommation est le fait surtout des plus jeunes, qui sont par ailleurs ceux qui se nourrissent le plus mal, sautant bien souvent les repas du matin et de midi.

\section{Conclusion}

Les représentations de Tahiti basées autour des notions d'abondance naturelle et de tradition d'accueil et d'hospitalité, font partie de l'imaginaire occidental, et sont également intériorisées par les populations locales. Si ces représentations ne portent pas à conséquence en matière sociale quand elles sont intégrées dans les spectacles folkloriques ou dans la communication touristique à l'égard des touristes internationaux comme des résidents, elles ont en revanche un impact important quand elles prennent la forme d'arguments visant à nier ou à minimiser l'existence même et les conditions de vie des groupes de population les plus précaires. C'est donc un rôle important que joue la question des représentations, fondées sur une facilité perçue d'accès aux ressources naturelles, et notamment dans les zones rurales de Tahiti ou dans les îles des autres archipels. Elle agit au niveau des différents acteurs de proximité chargés d'œuvrer auprès de ce groupe de population et permet ainsi de vérifier l'influence des représentations culturelles et sociales sur les attitudes et pratiques des sans-abri eux-mêmes suivant le modèle du jeu des interactions (Goffman 1971, 1974).

Au-delà de cette constatation, nous voudrions revenir sur la notion de désaffiliation qui nous paraît bien mieux s'appliquer aux sans-abri, en une sous-catégorie du concept plus général d'exclusion. En effet, le concept d'exclusion met plus l'accent sur des déterminants économiques que sociaux (et en ce sens est souvent assimilé à la "précarité » ou à la "pauvreté »), ce qui n'empêche pas pour autant l'existence de liens sociaux forts, notamment en Polynésie française. En effet, et malgré des situations économiques difficiles sinon précaires, on note dans les quartiers définis comme prioritaires par les pouvoirs politiques locaux, et notamment ceux qui font l'objet d'opérations de résorption de l'habitat insalubre (RHI), l'existence de réseaux de solidarité économique très forts, fondés sur l'autoconsommation et les échanges, vecteurs de recomposition sinon de pérennité de liens sociaux qui dépassent le strict lien de parenté. Malgré les quelques contre-exemples cités à propos des sans-abri, on ne peut pas pour autant considérer qu'ils sont inclus dans de tels réseaux sociaux et économiques, à commencer par les solidarités familiales si importantes en Polynésie française, dont le caractère défaillant paraît être une cause première à leur situation, avant même le facteur économique. C'est l'absence même de ce tissu relationnel, et notamment familial, qui nous paraît les définir, au-delà du constat de l'absence de logement et de la précarité de leur condition.

De plus, la diversité des situations rencontrées parmi cette population (et qu'illustrent notamment les écarts-types importants calculés dans l'analyse des données quantitatives de l'enquête) est souvent rassemblée sous un vocable unifiant, « le SDF » ou « le sans-abri », qui laisse croire à une homogénéité de pratiques de vie et de sociabilité. Or, nous ne partageons pas les propos de $\mathrm{P}$. Gaboriau (1993 : 15) sur une prétendue « culture de la rue » ou de la "place publique » partagée par tous les sans-abri autour de la mendicité, la consommation massive d'alcool et de drogues, et des modalités d'occupation des lieux publics, et qui dépasserait les particularités individuelles. L'esprit de survie et de " débrouille » nous semble ressortir d'une culture plus largement populaire, comme les « stratégies d'évitement » constatées auprès des sans-abri tahitiens relèvent d'une réaction face aux regards " des autres » et à la culpabilité et la honte ressenties, d'autant plus importantes que les autres sont des personnes connues : tactiques pour échapper au regard désapprobateur des passants qui peuvent faire

28. La Croix bleue est une forme de «contrat » écrit passé devant le pasteur de la paroisse protestante, en vertu duquel la personne s'engage devant Dieu à ne pas boire, et/ou fumer, et/ou jouer aux jeux d'argent, pendant une durée variable (de quelques mois à plusieurs années). Ce contrat remonterait au dix-neuvième siècle, et serait aujourd'hui encore précédé en début et en fin d'abstinence par une « beuverie monstre ». 
partie de la famille éloignée, réticence à bénéficier des aides matérielles mises à disposition par les structures associatives ou institutionnelles, refus des soins et du recours au médecin ou au psychologue...

Les représentations traditionnelles de Tahiti, une certaine forme «d'idéologie du retour » et des questions de catégorisation s'ajoutent en les renforçant aux représentations concernant les personnes vivant dans la rue, et ont une influence sur les attitudes et les comportements des acteurs institutionnels à même de mettre en œuvre sur le terrain les politiques sociales qui pourraient leur être destinées, de façon plus ou moins spécifique, rendant problématique leur prise en compte dans une optique de traitement social. Des expériences de "retour dans les îles" de personnes sans-abri ont ainsi été mises en œuvre, sans s'assurer au préalable des conditions de prise en charge et d'accompagnement dans les réseaux locaux sur place, et sans toujours prévoir d'aides en matériel agricole ou de pêche : les travailleurs sociaux notaient avec fatalité la présence de ces personnes dans les rues de Papeete de nouveau deux à trois mois après l'opération (« On envoie des personnes chez elles dans les îles, mais ensuite aucun suivi n'est fait: il n'y a qu'une assistante sociale pour tous les Tuamotu » selon un travailleur social).

Mais ces représentations ont de plus une influence sur les comportements mêmes des sans-abri : le fait de vivre dans la rue à Tahiti, bien plus difficile au quotidien que l'image que s'en fait le grand public, est en outre vécu sur le mode paradoxal et douloureux d'une libération pourtant facteur d'exclusion et de culpabilisation. Certains acteurs politiques ont conscience de la gravité et de la complexité d'un problème qui mêle des facteurs aggravants (conditions socio-économiques des familles dont est issue la grande majorité des sans-abri, handicaps psychologiques dus à des histoires personnelles souvent dramatiques...) et la faiblesse des moyens mis en œuvre (nombre relativement faible de travailleurs sociaux et de psychologues dans le secteur public pour un réel et indispensable accompagnement de ce public dans le temps, difficultés à rendre des terres disponibles du fait de l'indivision des propriétés foncières...). Mais les actions mises en place ces dernières années au profit des populations défavorisées ou « en situation d'exclusion » (logement social, protection sociale généralisée, dispositifs subventionnés d'aide à l'emploi ou à la formation ...) s'appliquent imparfaitement à cette population, qui n'est finalement pas prise en compte en tant que telle. Et les difficultés économiques que com- mence à connaître le pays, ainsi que l'instabilité politique récurrente depuis 2004, ne sont pas de nature à modifier cet état de fait. Les sans-abri tahitiens constituent donc la «part maudite » de l'abondance matérielle déversée ces quarante dernières années et la face obscure, de plus en plus visible, d'un mythe de l'abondance et de l'hospitalité familiale polynésiennes toujours vivace.

\section{BIBLIOGRAPHIE}

BeCKer Howard S., 1985. Outsiders. Études de sociologie de la déviance, Paris, Métailié.

Bourdieu Pierre, 1993. Comprendre, in Bourdieu Pierre (dir.), La misère du monde, Paris, Seuil, pp. 1388-1447.

Brousse Cécile, Bernadette RochÈre (de la) et Emmanuel Masse, 2002 (janvier). Hébergement et distribution de repas chauds. Qui sont les sans-domicile usagers de ces services?, INSEE Première 824.

CAstel Robert, 1991. De l'indigence à l'exclusion : la désaffiliation. Précarité du travail et vulnérabilité relationnelle, in Donzelot Jacques, Face à l'exclusion. Le modèle français, Paris, Éditions Esprit, pp. 137-168.

Cavaillet France et Milan Momic, 2004 (novembre). Une approche de la pauvreté par les budgets alimentaires en France, in CAVAILlet France (dir.), L'alimentation comme dimension spécifique de la pauvreté. Étude de la consommation alimentaire des populations défavorisées, rapport pour l'ONPES.

DAmon Julien, 2002. La question SDF. Critique d'une action publique, Paris, PUF, collection Lien social.

FAGES Jean, 1973. Les migrations humaines en Polynésie française, Cahiers de l'orstom, série Sciences humaines $X, 2 / 3$, pp. 289-293.

-, 1974. Migrations et urbanisation en Polynésie française, Cahiers de l'oRstom XI, 3/4, pp. 243-258.

Firdion Jean-Marie et Maryse MARPSAT, 2000. Les personnes sans domicile à Paris : caractéristiques, trajectoires et modes de vie, in J.-M. Firdion et $\mathrm{M}$. Marpsat (dir.), La rue et le foyer. Une recherche sur les sans-domicile et les mal-logés dans les années 1990, INED/PUF, pp. 257-285.

Fleuret Sébastien, Christian PIHET et Djemila ZeNEIDI-Henry, 2004. Connaissance des publics et de leurs parcours dans les structures d'accueil et d'hébergement d'urgence en Pays de la Loire, synthèse du rapport Unité mixte de recherche du CNRS 6590 Espaces géographiques et sociétés.

GABORIAU Patrick, 1993. Clochard, Paris, Julliard.

GofFMAN Erving, 1971. La mise en scène de la vie quotidienne. Tome 2 : les relations en public, Paris, Minuit. 
_, 1974. Les rites d'interaction, Paris, Minuit.

_, 1975. Stigmates. Les usages sociaux des handicaps, Paris, Minuit.

Institut de statistiQues de Polynésie FRAnÇAise, 2005. Pauvreté relative en Polynésie, l'approche monétaire, Papeete, Points forts 3/2005, 8 p.

_, 2007. Premiers résultats du recensement général de la population, Papeete, document de présentation des résultats.

Lockwood Victoria S., 1980. Development and Return Migration to Rural French Polynesia, International Migration Review 24 2, pp. 347-371.

_, 2002. Poverty in Paradise: development and relative income poverty in rural Tahitian society, Human Organization 61, 3, Society for Applied Anthropology.

Moreau de Bellaing Louis et Jacques Guillou, 1995. Les Sans Domicile Fixe. Un phénomène d'errance, Paris, L'Harmattan, 270 p.
PAUGAm Serge, 1991. La disqualification sociale. Essai sur la nouvelle pauvreté, Paris, Presses universitaires de France, collection Quadrige.

RochÈre (de la) Bernadette, 2003. Les sans-domicile ne sont pas coupés de l'emploi, INSEe Première 925.

Serra Mallol Christophe, 2005. Tahiti : du culte au mythe de l'abondance, Journal de la Société des Océanistes 120-121, pp. 149-156.

-, 2007. Changement social et traditions alimentaires. Approche socio-anthropologique de l'alimentation à Tahiti, Punaauia, Université de la Polynésie française, thèse de doctorat en anthropologie, 2 tomes.

-, 2008. Les SDF de l'agglomération de Papeete, Papeete, Contrat urbain de cohésion sociale de l'agglomération de Papeete, rapport de présentation détaillée des résultats.

ZeneIDI-Henry Djemila, 2002. Les SDF et la ville. Géographie du savoir-survivre, Paris, Bréal, Collection d'Autre Part. 